Pacific Journal of Mathematics

MAXIMUM TERM OF A POWER SERIES IN ONE AND 


\section{MAXIMUM TERM OF A POWER SERIES IN ONE AND SEVERAL COMPLEX VARIABLES}

\section{J. Gopala Krishna}

In this paper we discuss results leading to a description of an algebraic structure constituted by power series with the same maximum term in several complex variables, and use the description to estimate the maximum modulus of any of the series in terms of its maximum term and its central index, and obtain some useful asymptotic relations. We observe that certain crucial Valiron-type theorems, in the case of several variables, are by no easy or routine means reached through Valiron-type techniques.

The relations among the maximum term, the central index and the maximum modulus of a power series are extensively studied in the case of a single complex variable (see, e.g., [7]-[9]). While a number of Wiman-type theorems are extended to the case of several complex variables (see [1], [4] and their bibliographies) we find only some unsatisfactory attempts made to extend the Valiron-type results to the case of entire power series in two complex variables (see §6). Analogous to the case of one variable the Valiron-Wiman-type results contribute in their own way to the study of partial differential equations, and to obtain some such analogues (e.g., of [5], [6]) we need relations between the growths of the maximum term and the central index of a multiple power series along with certain Wiman-type theorems stronger than the ones available at present. In this paper, however, we limit ourselves to some Valiron-type results and attempt a systematic presentation, which in particular enables us to indicate certain open questions.

1. Notation. We write $\mathscr{C}^{k}$ for the cartesian product of the field of complex numbers with itself $k$ times and indicate its elements (points): $\left(z_{1}, z_{2}, \cdots, z_{k}\right),\left(\left|z_{1}\right|, \cdots,\left|z_{k}\right|\right),\left(r_{1}, \cdots, r_{k}\right),\left(n_{1}, \cdots, n_{k}\right)$ etc. by their corresponding unsuffixed symbols $z,|z|, r, n$, etc., when it is easy to understand from the context. Throughout $\Omega=\Omega_{k}$ stands for a nonempty open complete $k$-circular region in $\mathscr{C}^{k}$ (see $\S 3,3$ of [3]) with centre at $\overline{0}=(0,0, \cdots, 0)$, the zero element of $\mathscr{C}^{k}$. We write

$$
|\Omega|=[r: r=|z| \text { for some } z \in \Omega]
$$

and

$$
\Omega^{+}=\left[r: r \in|\Omega|, \text { no } r_{j}=0\right],
$$


and regard these as subsets of the $k$-dimensional euclidean space $\mathscr{E}^{k}$. In connection with any $r, s \in \mathscr{E}^{k}$ we say (i) that $r \leqq s$ or $s \geqq r$, if and only if $r_{j} \leqq s_{j}$ for $1 \leqq j \leqq k$, (ii) that $r<s$ or $s>r$, if and only if $r \leqq s$ but $r$ is not $=s$, and (iii) that $r \ll s$ or $s \gg r$, if and only if $r_{j}<s_{j}$ for $1 \leqq j \leqq k$.

Throughout $\mathscr{F}=\mathscr{F}(\Omega)$ stands for the family of all power series with centre at 0 and absolutely convergent in $\Omega$. Under conventions similar to those for indicating the elements of $\mathscr{C}^{k}$ we indicate $A \in \mathscr{F}$ by

$$
A(z)=\sum_{n \in \mathscr{N}} a_{n} z^{n}
$$

where (always)

$$
\begin{aligned}
\mathscr{N}=\mathscr{N}_{k} & =\left[n: n \in\left|\mathscr{C}^{k}\right|, \text { each } n_{j} \text { is a rational integer }\right], \\
z^{n} & =z_{1}^{n_{1}} z_{2}^{n_{2}} \cdots z_{k}^{n_{k}}\left(z_{j}^{0}=1 \text { even if } z_{j}=0\right) .
\end{aligned}
$$

Corresponding to an $A \in \mathscr{F}$ we define the functions (mappings): the maximum term $\mu=\mu\{A\}$, the central index $\nu=\nu\{A\}=\left(\nu_{1}, \cdots, \nu_{k}\right)$ and the maximum modulus $\mathscr{C}=\mathscr{C}\{A\}$ on $|\Omega|$ by

$$
\begin{aligned}
\mu(r) & =\mu(r, A)=\max _{n \in \mathscr{r}}\left(\left|a_{n}\right| r^{n}\right), \\
\nu_{j}(r) & =\nu_{j}(r, A)=\left\{\begin{array}{l}
\max \left[n_{j}:\left|a_{n}\right| r^{n}=\mu(r)\right], \text { if } \mu(r)>0, \\
0, \text { if } \mu(r)=0, \text { for } 1 \leqq j \leqq k,
\end{array}\right. \\
\mathscr{C}(r) & =\mathscr{L}(r, A)=\max _{|z|=r}|A(z)| .
\end{aligned}
$$

We say that a mapping $f$ with domain $D$ in a euclidean space and also with range in a euclidean space is increasing (in $D$ ), if and only if $f(r) \leqq f(s)$ for any $r, s \in D$ such that $r \leqq s$.

2. Fundamental properties of $\mu$ and $\nu$. Throughout this section we work with the same power series $A \in \mathscr{F}$. We start with

THEOREM 2.1. Let $p, r \in|\Omega|$ and let $\mu(p)$ and $\mu(r)$ be both positive. Then the line integral,

$$
I=\int_{p}^{r} \sum_{j=1}^{k} \frac{\nu_{j}(x)}{x_{j}} d x_{j}
$$

taken over any connected polygon in $|\Omega|$ with sides parallel to the axes and from $p$ to $r$, (i) exists, (ii) is independent of the polygon and (iii) is such that

$$
\log \mu(r)=\log \mu(p)+I .
$$

We need three lemmas. 
Lemma 2.2. Let $k>1$. Corresponding to any $r \in\left|\mathscr{C}^{k}\right|$, let $\dot{r}=\dot{r}^{(j)}$ denote the formal expression $\left(r_{1}, \cdots, r_{j-1}, \cdot, r_{j+1}, \cdots, r_{k}\right)$ and let $|\Omega|_{\dot{r}}$ denote the section of $|\Omega|$ corresponding $\dot{r}$, i.e., the set $\left[r_{j}\right.$ : $r \in|\Omega|$ ]. Let $|\Omega|_{\dot{r}}$ be nonempty. Let $\mu_{\dot{r}}$ and $\nu_{j \dot{r}}$ be respectively the sections of $\mu$ and $\nu_{j}$ corresponding to $\dot{r}$, defined on $|\Omega|_{\dot{r}}$ by $\mu_{\dot{r}}\left(r_{j}\right)=$ $\mu(r)$ and $\nu_{j \dot{r}}\left(r_{j}\right)=\nu_{j}(r)$.

Then $\mu_{\dot{r}}$ and $\nu_{j \dot{r}}$ are respectively the maximum term and the central index of the power series in one variable $\left(z_{j}\right)$ converging in $|\Omega|_{\dot{r}}$ viz.,

$$
\sum_{n \in r_{1}} \mu_{n_{j}} z_{j}^{n_{j}}
$$

where

$$
\mu_{n_{j}}=\mu_{r_{n_{j}}}=\max _{\left(n_{1}, \cdots, n_{j-1}, n_{j+1}, \cdots, n_{k}\right) \in \mathscr{N}_{k-1}}\left(\left|a_{n}\right| r_{1}^{n_{1}} \cdots r_{j-1}^{n_{j-1}} r_{j+1}^{n_{j+1}} \cdots r_{k}^{n_{k}}\right) .
$$

Proof of 2.2. After a complete statement the lemma is a straight forward verification. To see the idea of the lemma more quickly one might consider the special case where $k=2$ and look upon $A$ as a double series arranged in rows and columns in a "natural manner".

The following two lemmas are well-known (see, e.g. [7]). They however admit simple proofs (with no appeal to geometric intution), which we briefly indicate.

Lemma 2.4. Let $k=1$. Then (i) $\nu$ is increasing and right continuous and its discontinuities form a set discrete in $|\Omega|$; (ii) $\mu$ is increasing and continuous in $|\Omega|$.

Proof of 2.4. (ii) is a simple consequence of (i), which is easily proved (see also (2.6), (2.10), (2.14)).

Lemma 2.5. Let $k=1$. Then Theorem (2.1) holds in this case.

Proof of (2.5). It is sufficient to consider the case $p<r$, which we do. By (2.4) (i) there exists a finite dissection of the closed interval $[p, r]$ specified by $p=d_{0}<d_{1}<\cdots<d_{m}=r$ such that $\nu$ is continuous in each of the open intervals $\left(d_{j}, d_{j+1}\right)$. Now by $(2.4), \mu$ does not vanish in $[p, r]$ and for $j=0,1, \cdots, m-1$,

$$
\mu\left(d_{j+1}\right)=\mu\left(d_{j+1}-0\right)=\left|a_{\nu\left(d_{j}\right)}\right| d_{j+1}^{\nu\left(d_{j}\right)},
$$

which proves the result with $d_{j}, d_{j+1}$ respectively instead of $p, r$ and hence proves (2.5) itself. 
Proof of 2.1. By (2.2) and (2.5), (i) and (iii) hold in case the polygon under consideration is a straight line segment parallel to one of the axes (say to the $j$ th). The proof of (i) and (iii) may now be completed by using induction on the number of the sides of the polygon. (ii) is implied by the rest of the theorem.

We also require some extensions of (2.4) for $k>1$, with which we shall be concerned in the rest of this section. These extensions need a different approach and in particular we start making use of the openness of $\Omega$ (see (2.14)).

THEOREM 2.6. $\mu$ is (i) increasing and (ii) continuous in $|\Omega|$.

(i) of (2.6) is obvious. Before considering (ii) it is convenient to prove a lemma, which is required a few more times later.

Lemma 2.7. Let $r \in|\Omega|$ and $\mu(r)>0$. Then there exists a neighbourhood $V$ of $r$ w.r.t. $|\Omega|$ and a finite subset $\mathscr{L}$ of $\mathscr{N}$, such that for $t \in V$,

$$
\mu(t)=\max _{n \in \mathscr{L}}\left(\left|a_{n}\right| t^{n}\right)>\max _{n \in \mathscr{S}-\mathscr{S}}\left(\left|a_{n}\right| t^{n}\right) .
$$

Proof of 2.7. Since $\Omega$ is open in $\mathscr{C}^{k}$ there exists an $s \in|\Omega|$ such that $s \gg r$. It is easily seen that $\mu(r / 2)=\mu\left(r_{1} / 2, \cdots, r_{k} / 2\right)$ is also positive and hence there exists a finite set $\mathscr{L} \subseteq \mathscr{N}$ such that $\left|a_{n}\right| s^{n}<\mu(r / 2)$ for $n \in \mathscr{N}-\mathscr{L}$, which by virtue of the obvious (i) of (2.6) implies the lemma, when we take $V=[t: r / 2 \leqq t \leqq s]$.

Proof of 2.6. By virtue of (2.7) we need only prove the continuity of $\mu$ at points $r \in|\Omega|$, where $\mu(r)=0$. Let $r \in|\Omega|, \mu(r)=0$ and $\delta$ be a positive real number. As in the proof of (2.7), there exists an $s \in|\Omega|$ and a finite subset $\mathscr{L}=\mathscr{L}(\delta)$ of $\mathscr{N}$, such that $r \ll s$ and $\left|a_{n}\right| s^{n}<\delta$ for $n \in \mathscr{N}-\mathscr{L}$. This together with the fact that $\max \left[\left|a_{n}\right| t^{n}: n \in \mathscr{L}\right]$ defines a function continuous and vanishing at $t=r$, completes the proof.

It is not true that $\nu$ is increasing for $k>1$, as is shown by

ExAmple 2.8. Let $k>1, \Omega=\mathscr{C}^{k}$ and $A$ be the power series of $\mathscr{F}$, which converges at each $z \in \mathscr{C}^{k}$ to $1-k+\sum_{j=1}^{k} \exp z_{j}$. Then

$$
\nu_{j}(r)=\left[r_{j}\right] \text { or } 0,
$$

according as $r_{j}=$ or $<\max \left\{r_{1}, \cdots, r_{k}\right\}$ In particular $\nu(1, \cdots, 1)=$ $(1, \cdots, 1)$, while $\nu(1.5,1.55,1.555, \cdots)=(0,0, \cdots, 0,1)$. 
However one might consider different extensions of the fact that $\nu$ is increasing for $k=1$. We state, mainly for later use, the following obvious consequence of (2.2) and (2.4).

COROLLARY 2.9. $\nu_{j}$ is increasing and right continuous in the $j$ th variable $\left(i, e .\right.$, the $\nu_{j \dot{r}}$ of $(2.2)$ is increasing and right continuous in its domain), for $1 \leqq j \leqq k$.

We next prove

Theorem 2.10. Let $A$ be not identically zero and let $D$ denote the set of all discontinuities of $\nu$ in $|\Omega|$. Then there exists a finite or countable family of sets $D_{j}, j \in$ some index set $J$ such that

(i) $D=\bigcup_{j \in J} D_{j}$,

(ii) each $D_{j}$ is closed w.r.t. $|\Omega|$,

(iii) each $D_{j}$ is a subset of a hyper-surface of the form $[r: r \in|\Omega|$, $\left.r^{m}=\alpha\right]$, where $m_{1}, \cdots, m_{k}$ are rational integers and $\alpha$ is a real number $\geqq 0$ (and hence each $D_{j}$ is of at most real dimension $k-1$ ),

(iv) any compact subset of $|\Omega|$ in which $\mu$ does not vanish has a nonempty intersection with at most a finite number of the sets $D_{j}$; further

( v) $D$ itself is a nowhere dense closed set w.r.t. $|\Omega|$ and

(vi) $\left|a_{\nu(r)}\right| r^{\nu(r)}=\mu(r)$ for $r \in|\Omega|-D$.

We need two lemmas.

LEMMA 2.11. Let $A$ be not identically zero. Let $S$ denote the set of all $r \in|\Omega|$ at which $\mu(r)$ is attained by more than one term of the series $\sum_{n \in \mathscr{N}}\left|a_{n}\right| r^{n}$ and let

$$
S(m, n)=\left[r: r \in|\Omega|,\left|a_{m}\right| r^{m}=\left|a_{n}\right| r^{n}=\mu(r)\right] .
$$

Then (i) through (vi) of (2.10) hold with $S$ instead of $D$ and $\{S(m, n)(m \neq n)\}$ instead of $\left\{D_{j}, j \in J\right\}$.

Proof of 2.11. The analogues of (i), (ii), (iii) and (vi) of (2.10) are obvious.

By Lemma 2.7, to each $r \in|\Omega|$ at which $\mu(r)>0$, there corresponds a neighbourhood $V$ of $r$ suct that only a finite number of the sets $S(m, n)(m \neq n)$ have a nonempty intersection with $V$. The HeineBorel property of a compact set now implies the analogue of (iv) of (2.10).

The foregone discussion enable us to conclude that $S \cap \Omega^{+}$is nowhere dense in $|\Omega|$ and hence so is $S$. To complete the proof at 
this stage we need only show that $S$ is closed w.r.t. $|\Omega|$. Let $r \in|\Omega|$ and be a limit point of $S$. $r \in S$ obviously, if $\mu(r)=0$. Let $\mu(r)>0$. By the continuity of $\mu$ there exists a compact neighbourhood $P$ of $r$ w.r.t. $|\Omega|$ in which $\mu$ does not vanish. Hence by the analogues of (ii) and (iv) of (2.10), it follows that $P \cap S$ is closed w.r.t. $|\Omega|$, which in particular implies that $r \in S$.

Lemma 2.12. Let $A$ be not identically zero. Then the sets $D$ and $S$ introduced respectively in (2.10) and (2.11) are identical.

Proof of 2.12. Let $r \in|\Omega|$. We first consider the case: $\mu(r)>0$. Let us assume that $r \in S-D$. Now there exists a neighbourhood $V$ of $r$ in $|\Omega|$ in which $\mu$ does not vanish and $\nu$ is a constant, say $=m$. Hence by (2.6) and (2.11), $\mu(t)=\left|a_{m}\right| t^{m}$ for all $t \in V$. But since $r \in S$, there exists an $n \neq m$ such that $\left|a_{n}\right| r^{n}=\mu(r)$. This implies that for some $t \in V,\left|a_{n}\right| t^{n}>\left|a_{m}\right| t^{m}=\mu(t)$, which is impossible. Thus $r$ does not $\in S-D$. Using Lemma (2.7) we easily see that $r$ does no $\in D-S$. Thus $r \in S$, if and only if $r \in D$, in case $\mu(r)>0$.

Let us finally consider the case when $\mu(r)=0$. Now already $r \in S$, and to complete the proof we need only show that $r \in D$. Since $\mu(r)=0$, we also have $\nu(r)=\overline{0}$ and $a_{\overline{0}}=0$. We now assert that $r \in D$, for otherwise $\mu(t)=0$ for $t$ in a neighbourhood of $r$ w.r.t. $|\Omega|$, which is impossible since $\mu$ does not vanish in $\Omega^{+}$.

Proof of 2.10. (2.11) and (2.12) imply (2.10).

REMARK 2.13. Example (2.8) shows that for $k>1$, (vi) of (2.10) is not the best of its kind, although it is not true with $|\Omega|$ instead of $|\Omega|-D$.

REMARK 2.14. It is not clear whether we have obtained a rich collection of the properties of $\nu$ adequate enough to characterize it as a mapping when $k>1$. Further the techniques of this section have their own limitations, if one chooses to drop the openness of $\Omega$. For example, in such a general case, we would only be in a position to say, instead of Theorem (2.6) (ii), that $\mu$ is continuous w.r.t. any interval set $[t: r \leqq t \leqq s]$, where $r, s \in|\Omega|$. However our results may be easily generalized to the useful case in which $\Omega$ is a complete $k$ circular region and is a finite union of sets of which is open in $\mathscr{C}^{k}$ or some one of its axial subspaces (cf. $\S 3,3$ of [3]).

3. Algebraic structure of power series with the same maximum term. Throughout this section $\mu$ stands for the maximum term (function) of some power series of $\mathscr{F}$ and $\mathscr{K}=\mathscr{K}(\mu)$ stands for 
the partially ordered family of all series of $\mathscr{F}$ with maximum term $\mu$, where the strict partial order ' $A$ less than $B^{\prime}$ (' $B$ greater than $A^{\prime}$ ) for two elements $A, B \in \mathscr{K}$ is specified by $A<B(B>A)$, if and only if $\left|a_{n}\right| \leqq\left|b_{n}\right|$ for all $n \in \mathscr{N}$, with strict inequality for some $n$. We first prove the important

THEOREM 3.1. $\mathscr{K}$ admits maximal elements and admits only one such element $G=G\{\mathscr{K}\}$ with nonnegative real coefficients, given by

$$
G(z)=G(z, \mathscr{K})=\sum_{n \in \mathcal{r}} g_{n} z^{n},
$$

where

$$
g_{n}=\inf _{\alpha \in \Omega^{+}}\left[\mu(\alpha) / \alpha^{n}\right]=\inf _{\alpha \in\left[\alpha: \alpha \in|\Omega|, \alpha^{n}>0\right]}\left[\mu(\alpha) / \alpha^{n}\right], \text { for } n \in \mathscr{N} .
$$

Proof of 3.1. The equivalence of the two alternative expressions for each of the $g_{n}$ 's is implied by (2.6).

Let $z \in \Omega$. There exists an $\alpha \in \Omega^{+}$such that $|z| \ll \alpha$. Now

$$
\left|g_{n} z^{n}\right| \leqq \mu(\alpha)\left(|z|^{n} / \alpha^{n}\right), \text { for } n \in \mathscr{N}^{\prime} \text {. }
$$

Hence $G$ is absolutely convergent in $\Omega$.

(3.2) also shows that $\left|g_{n} z^{n}\right| \leqq \mu(|z|)$ for $n \in \mathscr{N}$ ("by making $\alpha \rightarrow|z|+\overline{0}$ "), so that

$$
\mu(|z|, G) \leqq \mu(|z|) \text {. }
$$

Now let $A \in \mathscr{K}$. For any $n \in \mathscr{N}$ and any $\alpha \in \Omega^{+}$we have: $\left|a_{n}\right| \alpha^{n} \leqq \mu(\alpha)$ or $\left|a_{n}\right| \leqq \mu(\alpha) / \alpha^{n}$, which implies that

$$
\left|a_{n}\right| \leqq g_{n}, \text { for } n \in \mathscr{N} \text {. }
$$

Hence

$$
\mu(|z|)=\mu(|z|, A) \leqq \mu(|z|, G),
$$

which together with (3.3) implies that $G \in \mathscr{K}$. Also the proof is complete since (3.4) holds for all $A \in \mathscr{K}$.

We now turn to the minimal elements of $\mathscr{K}$, which signify the possible gaps in a power series with an assigned maximum term. We need $r \in|\Omega|$.

TheOREM 3.5. Let $A, B \in \mathscr{K}$. Then $\nu(r, A)=\nu(r, B)$ for all

Proof of 3.5. By Theorem (2.1) and Corollary (2.9) the directional derivative of $\log \mu(r)$ in the direction of the positive $j$ th axis exists 
at all $r \in|\Omega|$, where $\mu(r)>0, r_{j}>0$, and is equal to $\nu_{j}(r, A) / r_{j}=$ $\nu_{j}(r, B) / r_{j}$, which by Corollary (2.9) implies that $\nu_{j}(r, A)=\nu_{j}(r, B)$ for all $r \in|\Omega|$ (the case when $\mu(r)=0$ being trivial).

Convention 3.6. Here after we write $\nu=\nu\{\mu\}$ for the common central index of the series of $\mathscr{K}(\mu)$. We now proceed to

THEOREM 3.7. $\mathscr{K}$ admits minimal elements and admits only one minimal element with nonnegative real coefficients.

Proof of 3.7. Let $E$ be the set of all points of continuity of $\nu$ in $|\Omega|$. Let

$$
s_{n}=\left\{\begin{array}{l}
g_{n}(\text { of }(3.1)), \text { if } n=\mathcal{\nu}(r) \text { for some } r \in E, \\
0 \text { for all other } n \in \mathscr{N} .
\end{array}\right.
$$

Since $G$ is absolutely convergent in $|\Omega|$, so does the series $S$ given by

$$
S(z)=\sum_{n \in \mathscr{S}} s_{n} z^{n}
$$

Now for any $r \in E$ and any $A \in \mathscr{K}$, by Theorem (2.10) (vi), $\nu(r)=$ $\nu(r, A)=\nu(r, G)=\nu(r, S)$ and

$$
\mu(r, S)=s_{\nu(r)} r^{\nu(r)}=\mu(r, G)=\mu(r)=\left|a_{\nu(r)}\right| r^{\nu(r)},
$$

which by virtue of (2.6) and (2.10) (v) imply that $\mu(r, S)=\mu(r)$ for all $r \in|\Omega|$, which means that $S \in \mathscr{K}$.

Finally (3.8) together with the fact that $\nu(E)=\nu\left(E \cap \Omega^{+}\right)$implies that

$$
S_{n} \leqq\left|a_{n}\right| \text { for } n \in \mathscr{N}, A \in \mathscr{K}
$$

which completes the proof.

REMARK 3.9. Our discussions of Theorems (3.1) and (3.7) further lead to the fact that the partially ordered subfamily of $\mathscr{K}$ consisting of all its series with nonnegative real coefficients is a distributive lattice closed for arbitrary unions and arbitrary intersections.

REMARK 3.10. In the case where $k=1$, Valiron [7] carried out his estimations using a characterization of the maximal element $G$ of $\mathscr{K}$ in terms of $\nu$, which is not available when $k>1$ (see $\S 6$ ). Also when $k=1$ we can say that $A$ itself is a maximal element of the $\mathscr{K}(\mu)$ determined by it, if and only if $\left|a_{n+2} a_{n}\right| \leqq\left|a_{n+1}\right|^{2}$ for $n \in \mathscr{N}_{1}$. We have no analogue of this for $k>1$, although Theorem (3.1) readily implies 
Corollary 3.11. If $A$ is the maximal element of the $\mathscr{K}(\mu)$ determined by it, then

$$
\left|a_{n+2(j)} a_{n}\right| \leqq\left|a_{n+1(j)}\right|^{2},
$$

for $n \in \mathscr{N}, 1 \leqq j \leqq k$, where $t(j)(t=1,2)$ stands for the element of $\mathscr{V}^{\circ}$ with $t$ at the jth place and zeros at others.

4. Estimates for $\mathscr{C l}$ in terms of $\mu$ and $\nu$. Throughout this section $A \in \mathscr{F}$ and $G$ is the maximal element, with nonnegative real coefficients (see Theorem (3.1)), of $\mathscr{K}\{A\}=\mathscr{K}(\mu)$, where $\mu$ is the maximum term of $A$.

THEOREM 4.1. Let $r \in|\Omega|$. Let $p \in\left|\mathscr{C}^{3 k}\right|$ and be such that $p \gg 1$, while $p r=\left(p_{1} r_{1}, \cdots, p_{k} r_{k}\right)$ still $\in|\Omega|$. Let

$$
N_{j}=\max _{r \leqq t \leqq p r} \nu_{j}(t), \text { for } 1 \leqq j \leqq k .
$$

Then

( i ) $\mu(r) \leqq \mu / C(r) \leqq G(r) \leqq \mu(r) \prod_{j=1}^{k}\left[N_{j}+p_{j} /\left(p_{j}-1\right]\right.$;

(ii) $\mu(r)=\mathscr{l}(r)$, if and only if the series $\sum_{n \in N} a_{n} r^{n}$ has at most one nonvanishing term;

(iii) the last relation in (i) is an equality, if and only if $\mu(r)=0$.

REMARK 4.2. Theorem (4.1) simultaneously extends two crucial results of Valiron for the cases (i) when $\Omega=\mathscr{C}^{11}$ (see Theorem 11, (2.10), Ch. II of [7]), and (ii) when $\Omega$ is bounded in $\mathscr{C}^{1}$ (see (9), (10), $\S 70$, Ch. IX of [8]). For example to get the result in case (i), we are only to choose $r$ such that $\nu(r)>0$ and $p=1+1 / \nu(r)$. In fact Theorem (3.1) provides the best upper estimate for $\mathscr{l}(r)$ in terms of $\mu$.

Proof of 4.1 ( i ). That $\mu(r) \leqq-\not C(r)$ follows easily from Taylor's Theorem and Cauchy's inequality (see [3]). That $\mathscr{C}(r) \leqq G(r)$ is obvious from Theorem (3.1). We thus need only consider the last relation.

Let $\mathscr{f}$ be the class of all subsets of the set $K$ of all positive integers $\leqq k$. Corresponding to each $J \in \mathcal{J}$, let us write

$$
\begin{aligned}
\mathscr{N}(J) & =\left[n: n \in \mathscr{N} ; n_{j} \geqq N_{j}, \text { if and only if } j \in J\right] ; \\
p(J) & =\left(\alpha_{1}, \cdots, \alpha_{k}\right), \text { where } \alpha_{j}=\left\{\begin{array}{l}
p_{j} \text { if } j \in J, \\
1 \text { otherwise },
\end{array}\right. \\
p(J) r & =\left(\alpha_{1} r_{1}, \cdots, \alpha_{k} r_{k}\right) .
\end{aligned}
$$

We shall also suppose that $\mu(r)>0$, as otherwise the theorem is trivial. By Theorem (3.1), 


$$
g_{n} \leqq \mu(p(J) r) /(p(J) r)^{n}
$$

for $n \in \mathscr{N}, J \in \mathscr{J}$, if $r^{n}>0$ (even if $r \notin \Omega^{+}$), and hence

$$
\begin{aligned}
G(r) & \leqq \sum_{J \in \mathscr{J}} \sum_{n \in \sim r(J)}\left[\mu\left(p(J) r /(p(J))^{n}\right]\right. \\
& =\sum_{j \in \mathscr{G}}\left[\mu(p(J) r) \prod_{j \in J} \frac{p_{j}^{1-N_{j}}}{p_{j}-1} \prod_{t \in K-J} N_{t}\right] .
\end{aligned}
$$

Since $\mu(r)>0$, by (2.6), $\mu(p(J) r)>0$, and hence by Theorem (2.1),

$$
\log \mu(p(J) r) \leqq \log \mu(r)+\sum_{j \in J} N_{j} \log p_{j},
$$

which together with (4.4) implies the last relation of (4.1)..(i).

Proof of 4.2 (ii). The result is essentially discussed when $k=1$ (see $\S 2$, Ch. I of [7]) and the technique of the proof may easily be extended to the case when $k>1$ (even if $r \in|\Omega|-\Omega^{+}$).

Proof of 4.2 (iii). The proof consists in observing that the first relation in (4.4) is not an equality when $\mu(r)>0$. Suppose this is not so. Then would follow (because of (4.3)) that $r^{n}>0$ for all $n \in \mathscr{N}$ and that (4.3) reduces to an equality, which implies (because of (2.6)) in particular that the series

$$
\sum_{n \in \mathscr{N}(K)} g_{n}(p r)^{n}
$$

is divergent, which contradicts (because of (3.1)) the fact that $p r \in|\Omega|$.

5. Asymptotic relations among $\mu, \nu$ and $\mathscr{C}$. In this section we throughout take $\Omega=\mathscr{C}^{k}$ and write $A$ to denote a nonconstant entire series of $\mathscr{F}$. We say that a real valued function $f$ defined outside a compact set in $|\Omega|$ is of finite order, if and only if there exist an $\alpha \in \Omega^{+}$and a positive real number $K$ such that $f^{+}(r) \leqq K r^{\alpha}$ asymptotically as $r \rightarrow \bar{\infty}=+\bar{\infty}=(+\infty, \cdots,+\infty)$, and we say that $A$ is of finite order, if and only if so is $\log ^{+} \mathscr{C}\{A\}$. Following RonkinFuks-ideas (cf. $\S 26,2$, Ch. V of [3]) we also talk of the hypersurface of systems of conjugate orders in the case of a function $f$ (or $A$ ) of finite order considered above. However it may be noted that our growth-indicators are rather associated wit Gol'dberg-order than with the growth-indicators introduced by Ronkin and Fuks (see Remarks (5.6), (5.7))

THEOREM 5.1. ( i ). The following three statements are equivalent:

(a) $A$ is of finite order;

(b) $\log ^{+} \mu\{A\}$ is of finite order; 
(c) each $\nu_{j}\{A\}(1 \leqq j \leqq k)$ is of finite order.

(ii) If $A$ is of finite order, the hypersurfaces of systems of conjugate orders of $A, \log ^{+} \mu\{A\}$ and $\theta\{A\}=\max _{1 \leqq j \leqq k} \nu_{j}\{A\}$ coincide.

Proof of 5.1 ( i ). For any $r, p \in|\Omega|$ such that $\mu(r)>0$ and $p \gg$ $(1, \cdots, 1)$, we get by $(2.9)$ that for $1 \leqq j \leqq k$,

$$
\nu_{j}(r) \leqq \frac{1}{\log p_{j}} \int_{r_{j}}^{p_{j} r_{j}} \nu_{j}\left(r_{1}, \cdots, r_{j-1}, x_{j}, r_{j+1}, \cdots, r_{k}\right) \frac{d x_{j}}{x_{j}},
$$

which by (2.1) and (2.6)

$$
\leqq[\log \mu(p r)-\log \mu(r)] p_{j} /\left(p_{j}-1\right),
$$

which shows that (b) implies (c).

It is easy to prove using (2.1), (2.6) and (2.9) that for a $d \in \Omega^{+}$ and any $r \in|\Omega|$ such that $\mu(r)>0$,

$$
\begin{aligned}
\log \mu(r) \leqq & \log \mu(d) \\
& +\sum_{j=1}^{k} \nu_{j}\left(r_{1}, \cdots, r_{j}, d_{j+1}, \cdots, \cdots, d_{k}\right) \log ^{+}\left(r_{j} / d_{j}\right),
\end{aligned}
$$

which (with the components of $d$ "fixed but chosen sufficiently large") shows that (c) implies (b).

By Theorem (4.1), (a) implies (b), and (b) and (c) together imply (a).

Proofs of 5.1 (ii). The proof involves no difficulties in the presence of our discussion for proving (5.1) (i).

THEOREM 5.2. Let $A$ be of finite order. Then $\log ^{+} \mu(r) \sim$ $\log ^{+} \mathscr{C}(r)$, as $r \rightarrow+\bar{\infty}$.

It is convenient to prove first

Lemma 5.3. Let $A$ be of finite order and be purely transcendental, i.e., there exists no $m \in \mathscr{N}$ such that $a_{n}=0$ for all $n \geqq m$. Then $\log ^{+} \mu(r) \sim \log ^{+} \mathscr{l l}(r)$, as $r \rightarrow+\bar{\infty}$.

Proof of 5.3. The hypothesis that $A$ is purely transcendental implies that

$$
[\log \mu(r)] / \log \left(r^{\alpha}\right) \rightarrow+\infty, \text { as } r \rightarrow+\infty \text {, }
$$

for any $\alpha \in\left|\mathscr{C}^{k}\right|$, while Theorems (4.1) and (5.1) imply the existence of an $\alpha \in\left|\mathscr{C}^{k}\right|$ such that

$$
\log \mathscr{C l}(r) \leqq \log \mu(r)+\log \left(r^{\alpha}\right)
$$


holds asymptotically as $r \rightarrow+\bar{\infty}$. Hence the lemma.

Proof of 5.2. We prove using induction on $k$, the number of variables. (5.3) implies the theorem when $k=1$ (see also Ch. II of [7]). Let us assume that $k>1$ and that the theorem holds in the case of $k-1$ instead of $k$ variables. By virtue of (5.3) it is sufficient to obtain the conclusion of the theorem for $k$ variables when there exists an $m \in V^{\prime}$ such that $a_{n}=0$ for all $n \geqq m$, and this we do. Now for $r \in|\Omega|$,

$$
\begin{aligned}
\mathscr{C l}(r) & \leqq \sum_{j=1}^{k}\left[\sum_{n \in,}\left|a_{n}\right| r^{n}\right] \\
& \leqq \sum_{j=1}^{k}\left[\sum_{t=0}^{m_{j}-1} H_{\jmath t}\left(\dot{r}^{(j}\right) r_{j}^{t}\right],
\end{aligned}
$$

where $\dot{r}=\dot{r}^{j j}=\left(r_{1}, \cdots, r_{\jmath-1}, r_{j+1} \cdots r_{k}\right)$ and $H_{j t}$ represents an entire power series with centre at $\overline{0}$ in $k-1$ variables such that $H_{j t}\left(\dot{r}^{\left(t_{j}\right.}\right) r_{j}^{t}$ represents a sub-series of $\sum_{n \epsilon},\left|a_{n}\right| r^{n}$, for $0 \leqq t<m_{j}, 1 \leqq j \leqq k$. In particular we have

$$
\mu_{j t}\left(\dot{r}^{(j)}\right) r_{\jmath}^{t} \leqq \mu(r), \quad \text { for } \quad r \in|\Omega|,
$$

where $\mu_{j t}$ is the maximum term of $H_{\jmath t}$, for $0 \leqq t<m_{j}, 1 \leqq j \leqq k$. By our induction hypothesis (and trivially if $H_{\jmath k}$ reduces to a constant) it follows that

$$
H_{j t}\left(\dot{r}^{(j)}\right) \leqq \mu_{j t}^{1+\hat{t}}\left(\dot{r}^{(j)}\right) \text {, for } 0 \leqq t<m_{j}, 1 \leqq j \leqq k, \delta>0 \text {, asymptotical- }
$$
ly as $\dot{r}^{(j)} \rightarrow+\bar{\infty}$ in $\left|\mathscr{f}^{k-1}\right|$. Hence by virtue of (5.4) and (5.5) we get that

$$
\mathscr{l l}(r) \leqq k\left(\sum_{j=1}^{k} m_{j}\right) \mu^{1+\delta}(r)
$$

asymptotically as $r \rightarrow+\bar{\infty}$, for any positive real number $\delta$, which together with (4.1) and the fact that $\mu(r) \rightarrow+\infty$ as $r \rightarrow+\infty$ completes the proof.

REMARK 5.6. For $k>1$, Theorem (5.2) would be false, if one chooses to interpret "of finite order" following Ronkin and Fuks (see $\S 26,2$, Ch. V of [3]), although the analogue of Theorem (5.1) would still be true. To disprove the analogue of (5.2) one may consider $A$ such that $A(z)=\exp z_{1}$ for all $z \in \mathscr{C}^{k}(k>1)$.

REMARK 5.7. In the presence of the discussions of this section it is easy to state and prove the analogues of (5.1) and (5.2) keeping Gol'dberg- order of $A$ in mind, which does not really depend on the fundamental domain used for defining it (see $\S 26,1$, Ch. V of [3]). 
Also one might try to generalise our considerations of this section to the case where $\Omega$ is not necessarily the whole of $\mathscr{C}^{k}$, so as to be able to present in particular a unified picture of the unbounded and the bounded cases of $\Omega \cong \mathscr{C}^{1}$ (see Ch. II of [7] and the indications of $\S 70$, Ch. IX of [8]).

6. Appendix. An extension of Valiron's theory to the case of entire power series in two complex variables is attempted by $\mathrm{S}$. K. Bose and Devendra Sharma [2]. They start with a series $C \in \mathscr{F}\left(\mathscr{G}^{22}\right)$ and state to have constructed using Valiron-type geometrical considerations, a maximal element $W$ of the $\mathscr{C}\{C\}$ determined by $C$, in the notation of our $\S 3$, whose coefficients admit a specific pattern (see (4.1)-(4.2) of [2], cf. Ch. II of [7]). They take $w_{0}^{-}=1$ and using the special forms of the coefficients of $W$ obtain their basic formulae connecting the $\mu$ and $\nu$ of $C$ ((4.3), (4.4) of [2]).

Apart from the fact that the treatment of Bose and sharma $(\S 4$ and $\S 5$ of [2]) needs cautious handling, their basic formulae under reference, in particular, are incorrect. We give one example $((6.1))$ of transcendental $C$ in the case of which the series $W$ (of the sort they need) does not exist and their basic formulae fail. We give another example ((6.2)) of $C$, which for itself has all coefficients positive and real but in the case of which again their basic formulae fail. However it may be noted that some of the asymptotic relations considered by them turn out to be correct (being included in our Theorems (5.1), (5.2)).

EXAmple 6.1. Let $C \in \mathscr{F}\left(\mathscr{C}^{2}\right)$ and be such that its sum $C(z)=$ $\exp \left(z_{1} z_{2}\right)$, for $z \in \mathscr{C}^{2}$. For this series (4.3) or (4.4) of [2] imply that $\mu(r)=+\infty$ particularly whenever $r \geqq(1.1)$, which is obviously false. This itself also shows that the type of $W$, Bose and Sharma require, does not exist in the present content. Obviously the "Hadamard Polyhedron" of $C$ [2] degenerates virtually to a space polygon. We would further assert that there can be no series (of $\mathscr{F}\left(\mathscr{C}^{2}\right)$ ) with the same maximum term as $C$, whose coefficients are all nonvanishing.

Let $A \in \mathscr{F}\left(\mathscr{C}^{2}\right)$ and let $\mu\{A\}=\mu\{C\}$. Now for any $n \in \mathscr{N}_{2}$,

$$
\begin{aligned}
\left|a_{n}\right| & \leqq \inf _{r_{1} r_{2}=n_{1}}\left[\mu(r) / r^{n}\right] \\
& =\inf _{r_{1} r_{2}=n_{1}}\left[r_{2}^{n_{2}-n_{1}} /\left(n_{1} !\right)\right] \\
& =0, \text { if } n_{2}<n_{1} .
\end{aligned}
$$

Hence and by symmetry $a_{n}=0$, whenever $n_{1} \neq n_{2}$, which establishes our assertion. With the help of the discussions of $\S 3$, it is also easily seen that $C$ is both a maximal and a minimal element of the $\mathscr{K}\{C\}$ determined by it. 
ExAmple 6.2. Let $C \in \mathscr{F}\left(\mathscr{C}^{2}\right)$ and be such that its sum $C(z)=$ $\exp \left(z_{1}+z_{2}\right)-z_{1} / 2-z_{2} / 2$, for $z \in \mathscr{C}^{2}$. Now all the coefficients of $C$ are real and positive, so that its "Hadamard Polyhedron" suffers no degeneracy. Still one might observe that the formulae (4.3) and (4.4) of [2] do not hold, in particular when $r_{1}=r_{2}=1$. In fact one encounters some defective notation in [2] such as writing $\nu_{j}\left(r_{j}\right)$ instead of our $\nu_{j}(r)(j=1,2)$, which may however be understood from the context.

\section{REFERENCES}

1. T. F. Bitlyan and A. A. Goldberg, The Wiman-Valiron theorems for entire functions of several complex variables, Vestnik Leningrad University No. 13, Ser. Math. Mech. Astr. 2 (1959), 27-41 (Russian).

2. S. K. Bose and Devendra Sharma, Integral functions of two complex variables, Compositio Mathematica 15 (1963), 210-226.

3. B. A. Fuks, Introduction to the theory of functions of several complex variables, Amer. Math. Soc., 1963.

4. F. I. Geče. Systems of entire functions of several variables with applications to the theory of differential equations, Izv. Akad. Nauk Armjan S.S.R., Ser Fiz Mat. Nauk 17 (1964), 17-46 (Russian).

5. S. M. Shah, Entire functions of bounded index, Proc. Amer. Math. Soc. (to appear) 6. - Entire functions satisfying a linear differential equation., J. Math. Mech. (to appear)

7. G. Valiron, General theory of integral functions., Chelsea Publishing Company, 1949.

8. - Fonctions analytiques, Presses Universitaires De France, 1954.

9. H. Wittich, Neuere Untersuchungen Über Eindeutige Analytische Functionen, Springer-Verlag, 1955.

Received March 19, 1968,

UNIVERSITY OF KENTUCKY 


\section{PACIFIC JOURNAL OF MATHEMATICS}

\section{EDITORS}

H. ROYDEN

Stanford University

Stanford, California

\author{
R. R. Phelps \\ University of Washington \\ Seattle, Washington 98105
}

J. DugundJI

Department of Mathematics

University of Southern California

Los Angeles, California 90007

RICHARD ARENS

University of California

Los Angeles, California 90024

\section{ASSOCIATE EDITORS}

E. F. BECKenbaCH

B. H. NEUMANN

F. WOLF

K. YoSHIDA

\section{SUPPORTING INSTITUTIONS}

\author{
UNIVERSITY OF BRITISH COLUMBIA \\ CALIFORNIA INSTITUTE OF TECHNOLOGY \\ UNIVERSITY OF CALIFORNIA \\ MONTANA STATE UNIVERSITY \\ UNIVERSITY OF NEVADA \\ NEW MEXICO STATE UNIVERSITY \\ OREGON STATE UNIVERSITY \\ UNIVERSITY OF OREGON \\ OSAKA UNIVERSITY \\ UNIVERSITY OF SOUTHERN CALIFORNIA
}

\author{
STANFORD UNIVERSITY \\ UNIVERSITY OF TOKYO \\ UNIVERSITY OF UTAH \\ WASHINGTON STATE UNIVERSITY \\ UNIVERSITY OF WASHINGTON \\ $\stackrel{*}{*} \stackrel{*}{*} \stackrel{*}{*}$ AMERICAN MATHEMATICAL SOCIETY \\ CHEVRON RESEARCH CORPORATION \\ TRW SYSTEMS \\ NAVAL WEAPONS CENTER
}

The Supporting Institutions listed above contribute to the cost of publication of this Journal, but they are not owners or publishers and have no responsibility for its content or policies.

Mathematical papers intended for publication in the Pacific Journal of Mathematics should be in typed form or offset-reproduced, double spaced with large margins. Underline Greek letters in red, German in green, and script in blue. The first paragraph or two must be capable of being used separately as a synopsis of the entire paper. It should not contain references to the bibliography. Manuscripts, in duplicate if possible, may be sent to any one of the four editors. Please classify according to the scheme of Math. Rev. 36, 1539-1546. All other communications to the editors should be addressed to the managing editor, Richard Arens, University of California, Los Angeles, California, 90024.

50 reprints are provided free for each article; additional copies may be obtained at cost in multiples of 50 .

The Pacific Journal of Mathematics is published monthly. Effective with Volume 16 the price per volume (3 numbers) is $\$ 8.00$; single issues, $\$ 3.00$. Special price for current issues to individual faculty members of supporting institutions and to individual members of the American Mathematical Society: $\$ 4.00$ per volume; single issues $\$ 1.50$. Back numbers are available.

Subscriptions, orders for back numbers, and changes of address should be sent to Pacific Journal of Mathematics, 103 Highland Boulevard, Berkeley, California, 94708.

PUBLISHED BY PACIFIC JOURNAL OF MATHEMATICS, A NON-PROFIT CORPORATION

Printed at Kokusai Bunken Insatsusha (International Academic Printing Co., Ltd.), 7-17, Fujimi 2-chome, Chiyoda-ku, Tokyo, Japan. 


\section{Pacific Journal of Mathematics \\ Vol. 29, No. $3 \quad$ July, 1969}

Herbert James Alexander, Extending bounded holomorphic functions from certain subvarieties of a polydisc ...................... 485

Edward T. Cline, On an embedding property of generalized Carter

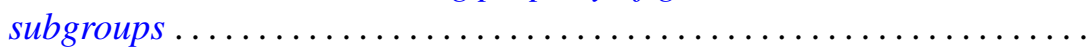

Roger Cuppens, On the decomposition of infinitely divisible characteristic functions with continuous Poisson spectrum. II ...............

William Richard Emerson, Translation kernels on discrete Abelian

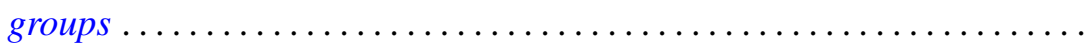

Robert William Gilmer, Jr., Power series rings over a Krull domain ....... 543

Julien O. Hennefeld, The Arens products and an imbedding theorem ...... 551

James Secord Howland, Embedded eigenvalues and virtual poles ........ 565

Bruce Ansgar Jensen, Infinite semigroups whose non-trivial homomorphs are all isomorphic .............................. 583

Michael Joseph Kascic, Jr., Polynomials in linear relations. II .......... 593

J. Gopala Krishna, Maximum term of a power series in one and several

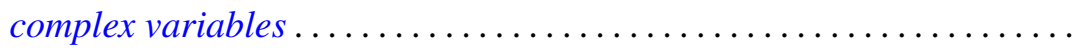

Renu Chakravarti Laskar, Eigenvalues of the adjacency matrix of cubic lattice graphs ...................................

Thomas Anthony Mc Cullough, Rational approximation on certain plane

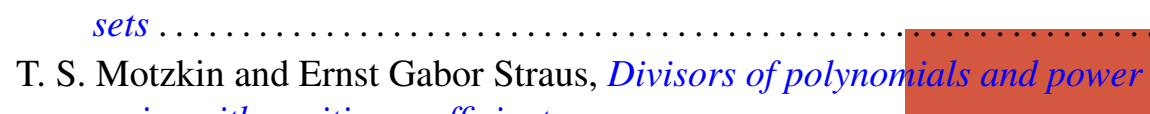
series with positive coefficients .

Graciano de Oliveira, Matrices with prescribed characteristic polynomial and a prescribed submatrix.

Graciano de Oliveira, Matrices with prescribed characteristic polynomial and a prescribed submatrix. II .

Donald Steven Passman, Exceptional 3/2-transitive permutation groups .................................

Grigorios Tsagas, A special deformation of the metric with no negative sectional curvature of a Riemannian space............

Joseph Zaks, Trivially extending decompositions of $E^{n}$ 